\title{
Measuring Immigration and Sexual Reproduction in Field Populations of Mycosphaerella graminicola
}

\author{
J. Zhan, C. C. Mundt, and B. A. McDonald
}

First and third authors: Department of Plant Pathology and Microbiology, Texas A\&M University, College Station 77843-2132; and second author: Department of Botany and Plant Pathology, Oregon State University, Corvallis.

Current address of B. A. McDonald: Phytopathology Group, Institute for Plant Sciences, Swiss Federal Institute of Technology, CH-8092 Zurich, Switzerland.

Accepted for publication 27 August 1998.

\begin{abstract}
Zhan, J., Mundt, C. C., and McDonald, B. A. 1998. Measuring immigration and sexual reproduction in field populations of Mycosphaerella graminicola. Phytopathology 88:1330-1337.

A field experiment was conducted to determine the relative contributions of immigration and sexual reproduction to the genetic structure of Mycosphaerella graminicola populations during the course of an epidemic. The genetic structure of $M$. graminicola populations sampled from wheat plots inoculated artificially with 10 isolates was compared with control plots infected naturally by airborne ascospores. Restriction fragment length

gametic equilibrium, findings consistent with random mating. The proportion of isolates in the inoculated plots with DNA fingerprints that differed from the 10 inoculated isolates increased from $3 \%$ in the early to 39 and $34 \%$ in the mid- and late season, respectively. The degree of gametic disequilibrium was higher in the mid-season than in the late-season population. By the end of the growing season, we estimate that $66 \%$ of the isolates in the inoculated plots were asexual progeny of the 10 inoculated isolates, $10 \%$ were immigrants, and $24 \%$ were sexual recombinants. The proportion of infections caused by ascospores increased over the growing season.
\end{abstract} polymorphisms (RFLPs) were used to test the randomness of associations among loci, and DNA fingerprints were used to identify clones. All isolates in the control plots had unique genotypes and RFLP loci were at
Additional keywords: epidemiology, gene flow, mating system, population genetics, Septoria tritici.
Migration and mating system may have profound effects on the genetic structure of fungal populations. Migration increases the gene diversity of local populations by introducing novel alleles from other populations. It also decreases genetic differentiation among populations by equalizing allele frequencies across populations. Knowledge of migration rate may help predict how fast novel genotypes with fungicide resistance, virulence genes, or both will spread among populations (22). Mating system affects the genotype diversity of populations, but it is not expected to affect gene diversity. Populations exhibiting largely asexual reproduction tend to have low genotypic diversity arrayed as a limited number of clonal lineages (15). As a result, asexual populations are expected to exhibit a significant degree of nonrandom association among unlinked loci (gametic disequilibrium) (25). In contrast, sexual populations are expected to exhibit a high degree of genotypic diversity and random associations (gametic equilibrium) among neutral loci as a result of the reassortment of unlinked genes during meiosis. For example, in Puccinia graminis f. sp. tritici, Burdon and Roelfs (4) found that populations undergoing sexual reproduction had more genotypes than those with strictly asexual reproduction. Roelfs and Groth (29) detected a low and continuous decline in race diversity over time in populations with asexual reproduction. Nonrandom associations between isozyme alleles and virulence genes were found in the asexual populations, but not in the sexual populations (4).

The relative impacts of migration, sexual reproduction, and asexual reproduction on the genetic structure of a fungal population will depend on features of its ecology and biology. Species dispersed by airborne spores are likely to exhibit higher rates and distances

Corresponding author: B. A. McDonald

E-mail address: bruce.mcdonald@ipw.agrl.ethz.ch

Publication no. P-1998-1022-02R

(C) 1998 The American Phytopathological Society of migration than species that produce only splash-dispersed spores. Environmental conditions are likely to affect the timing and rates of formation of sexual and asexual fruiting bodies (27).

Although it is known that many fungi exhibit a mixture of sexual and asexual reproduction in nature (17) and that migration among populations can be significant $(15,24)$, it is difficult to determine how migration interacts with sexual reproduction and asexual reproduction to affect the genetic structure of fungal populations. Statistical analyses of population subdivision $(34,35)$ and departures from gametic disequilibrium (6) in natural populations may provide indirect measures of gene flow and mating system parameters. For example, Brown and Wolfe (3) estimated that about $24 \%$ of the primary inoculum of Erysiphe graminis f. sp. hordei was ascospores by comparing gametic disequilibrium at two different times in the year. However, experiments with marked isolates ultimately are needed to make direct estimates of rates of migration and sexual recombination in field populations. These experiments are now possible given the availability of molecular markers that are sensitive enough to distinguish asexual progeny from novel genotypes and the statistical methods that can separate recombinants from immigrants.

Mycosphaerella graminicola (Fuckel) J. Schroeter ex Cohn (anamorph Septoria tritici Roberge ex Desmaz.) is a haploid fungus that causes Septoria tritici leaf blotch on wheat. This pathogen is distributed worldwide (9) and causes significant losses in many wheat-growing areas $(10,14,31)$. The incidence and economic significance of Septoria tritici leaf blotch have increased steadily since the 1960s as a result of the widespread and rapid replacement of local cultivars with early-maturing, semidwarf, higher-yielding cultivars that are susceptible to this pathogen (8). Changes in cultural practices $(8,16)$ and applications of phosphorus fertilizer (16) have also contributed significantly to the increased disease incidence.

The most economical method for controlling Septoria tritici leaf blotch is to use genetic resistance (36). Breeding of resistant culti- 
vars is impeded by the complex inheritance of resistance (28, $30,32,38)$ and by the positive association of resistance with late maturity and tall stature (8). Resistance in wheat cultivars may break down as a result of selection for corresponding virulence in the pathogen population (26). Both mating system and migration will significantly affect the response of the pathogen population to selection.

Previous studies indicated that sexual reproduction plays a significant role in the population genetics of M. graminicola (6) and in the epidemiology of the disease (33). The teleomorph has been identified in several countries (14). Ascospores produced by the teleomorph are dispersed by wind (16), and pycnidiospores produced by the anamorph are disseminated by rain splash (14). It was hypothesized that airborne ascospores originating from wheat stubble between seasons served as the primary inoculum at the start of the growing season, while secondary inoculum during the growing season was from splash-dispersed asexual pycnidiospores $(18,33)$. However, more recent studies indicated that ascospores originating from within the crop canopy may contribute to disease spread during the growing season $(12,13)$. The objective of this study was to quantify the relative contributions of immigration, sexual reproduction, and asexual reproduction to the genetic structure of M. graminicola populations through the course of a growing season.

\section{MATERIALS AND METHODS}

The experiment was conducted at the Oregon State University Botany and Plant Pathology Field Laboratory in Corvallis during the 1994-1995 winter wheat season. Field plots were arranged in a randomized complete block design with four replications. Wheat plots were planted in a checkerboard pattern with plots of barley (a nonhost for this $M$. graminicola population) separating each plot of wheat. Each replication contained three plots planted either to the wheat cultivar Madsen or Stephens or to a 50:50 mixture of 'Madsen' and 'Stephens'. Plots were $3.3 \mathrm{~m}$ (12 rows) $\times 5.3 \mathrm{~m}$ in size. The site used for this experiment was not planted to wheat in previous years, and the nearest wheat field was approximately $3 \mathrm{~km}$ away.

Ten isolates of M. graminicola having different DNA fingerprints were inoculated onto the wheat seedlings in November 1994. Five of these isolates, S1 to S5, were collected at Corvallis in the previous year from 'Stephens'. The other five isolates, M1 to M5, were collected at Corvallis in the previous year from 'Madsen'. Spores from the 10 isolates were mixed in equal proportion, and an aqueous suspension of one million spores per milliliter was sprayed onto three of the four replications using small, hand-carried sprayers. The fourth (control) replication was inoculated naturally by airborne ascospores. Samples consisting of 100 infected leaves were collected from the inoculated plots on 10 February, 3 April, and 2 June 1995, hereafter referred to as early, mid-, and late season, respectively. For the early- and late-season collections, infected leaves were sampled from all nine inoculated plots. In the mid-season, only the three inoculated plots of 'Stephens' were sampled. Sixty infected leaves were collected on 10 February 1995 from the control plots. In all cases, leaves were sampled from the inner eight rows of each plot. Each leaf was sampled from a different plant. The leaves were airdried at room temperature for 2 weeks before fungal isolations were made, as described previously (5).

DNA extraction, restriction enzyme digestion, Southern blotting, and hybridization. Total DNA from each isolate was extracted using cetyltrimethylammonium bromide, as described previously (19). Five micrograms of DNA from each isolate was digested with the restriction enzyme PstI. DNA fragments were separated by electrophoresis through $0.8 \%$ agarose Tris-borate-EDTA gels. The DNA fragments in the agarose gels were transferred to nylon membranes by alkaline capillary transfer. Afterward, the membranes were dried and stored in sealed plastic bags until needed. Nine anonymous DNA probes (pSTS192, pSTS43, pSTS2, pSTS197, pSTL53, pSTL31, pSTS14, pSTL10, and pSTL70) (19) were hybridized sequentially to the Southern blots. Probe pSTL70 is a DNA fingerprinting probe that hybridizes to a moderately repetitive DNA sequence dispersed across several chromosomes (20). The remaining eight probes hybridized to nine restriction fragment length polymorphism (RFLP) loci distributed across nine chromosomes (pSTS192 hybridizes to two unlinked loci [21]). The probes were radioactively labeled with $\mathrm{dCT}^{32} \mathrm{P}$ via nick translation. The labeled probes were allowed to hybridize to the membranes overnight at $60^{\circ} \mathrm{C}$ in a hybridization incubator. Following hybridization, the membranes were washed according to the manufacturer's instructions (Bio-Rad Laboratories, Hercules, CA) and exposed to $\mathrm{X}$-ray film at $-80^{\circ} \mathrm{C}$. The radioactive probes were stripped off the membranes after the films were developed, following the manufacturer's instructions (Bio-Rad Laboratories).

Data analysis. For the eight probes that hybridized to single loci, only alleles that could be scored unambiguously were included in the analysis. Isolates with identical DNA fingerprints were treated as individual members of the same clone (20), the products of asexual reproduction. Isolates with DNA fingerprints that matched 1 of the 10 inoculated isolates were excluded from analyses of gametic disequilibrium described below.

Analysis of variance on proportions of novel isolates were performed by SAS (SAS Institute, Cary, NC) using the general linear model. Before performing analysis of variance, proportions were transformed by taking natural logarithms. Comparisons of allele frequencies between control and inoculated plots were based on the contingency chi-squared test proposed by Everitt (7).

To determine if gametic disequilibrium existed in any collection of isolates, the significance of multilocus associations was measured with the method proposed by Brown et al. (2) as follows. Let $K$ be the number of loci that have different alleles (these are termed heterozygous loci even though $M$. graminicola is haploid) when two random gametes (for haploid organisms, gametes are equivalent to individuals) are compared at these loci; $p_{j i}$ and $p_{l k}$ are the gene frequencies of the $i$ th allele at the $j$ th locus and the $k$ th allele at the $l$ th locus, respectively. The observed variance of $K$ heterozygous loci will then be

$$
S_{k}^{2}=\sum h_{j}-\sum h_{j}^{2}+2 \sum_{j} \sum_{l>j} \sum_{i} \sum_{k}\left[2 p_{j i} p_{l k} D_{i k}^{j l}+\left(D_{i k}^{j l}\right)^{2}\right]
$$

in which $h_{j}$ is the single-locus gene diversity and $D_{i k}^{j l}$ is the gametic disequilibrium between the $i$ th allele at the $j$ th locus and the $k$ th allele at the $l$ th locus. Assuming that allelic distributions among loci are independent, the sampling variance of $S_{k}^{2}$ will be

$$
\operatorname{var}\left(S_{k}^{2} \mid \text { Ho is true }\right)=\left[\sum h_{j}-7 \sum h_{j}{ }^{2}+12 \sum h_{j}{ }^{3}-6 \sum h_{j}{ }^{4}+2\left(\sum h_{j}-\sum h_{j}{ }^{2}\right)^{2}\right] / n
$$

in which $n$ is the sample size. If the sampling distribution of $S_{k}^{2}$ approximates normality, the upper $95\left(L_{1}\right)$ and $99 \%\left(L_{2}\right)$ confidence limits for $S_{k}^{2}$ will be

$$
L_{1}=\sum h_{j}-\sum h_{j}{ }^{2}+1.98\left[\operatorname{var}\left(S_{k}^{2} \mid \text { Ho is true }\right)\right]^{1 / 2}
$$

and

$$
L_{2}=\sum h_{j}-\sum h_{j}^{2}+2.56\left[\operatorname{var}\left(S_{k}^{2} \mid \text { Ho is true }\right)\right]^{1 / 2}
$$

If the observed $S_{k}^{2}$ exceeds $L_{1}$ or $L_{2}$, the null hypothesis of independence between locus pairs will be rejected at $p=0.05$ and 0.01 levels of significance, respectively.

To determine which pairs of loci contributed to the overall disequilibrium detected by Brown's method, the significance of gametic disequilibrium between all pairwise combinations of RFLP loci was calculated with the methods proposed by Weir (37). A chisquared test for departures from gametic equilibrium across all $k$ 
alleles for the first locus and $l$ alleles for the second locus was formulated as

$$
\chi_{T}^{2}=\sum_{u=1}^{k} \sum_{v=1}^{l} \frac{n \hat{D}_{u v}^{2}}{\widetilde{P}_{u} \widetilde{P}_{v}}
$$

For loci that had significant disequilibrium, a test to determine which pairs of alleles at those loci exhibited nonrandom associations was formulated as

$$
\chi_{u v}^{2}=\frac{n \hat{D}_{u v}^{2}}{\widetilde{P}_{u}\left(1-\widetilde{P}_{u}\right) \widetilde{P}_{v}\left(1-\widetilde{P}_{v}\right)}
$$

in which $\widetilde{P}_{u}$ and $\widetilde{P}_{v}$ were the observed frequencies of alleles $u$ and $v$ for any two loci, $n$ was the sample size, and $\hat{D}_{u v}$ was the maximum likelihood estimate for the coefficient of disequilibrium between alleles $u$ and $v$. These chi-squared statistics had $(k-1)(l-1)$ degrees of freedom for locus-by-locus comparisons and 1 degree of freedom for allele-by-allele comparisons. For the locus-by-locus comparisons, rare allele pairs could cause a high value for the chisquared test because of a low expected number in the denominator, causing a statistically significant but spurious departure from random association. To diminish the effect of rare allele pairs, all alleles present at frequencies less than 0.10 were pooled into a single category.

Differentiating immigrants and recombinants. The proportion of novel genotypes resulting from sexual recombination among the inoculated isolates within the plot and resulting from immigration from outside the inoculated plots was estimated using absolute and relative probabilities for each novel genotype. Absolute probabilities were calculated based on both an immigration model and a sexual recombination model as follows. Let the frequency of allele $i$ at locus $j$ under the immigration (I) model and recombination $(\mathrm{R})$ model be

$$
p_{i j}^{\mathrm{I}} \text { and } p_{i j}^{\mathrm{R}}
$$

respectively. Assume loci are neutral (23) and populations are random mating (6). The probability that a genotype will then contain the $i$ th allele at the $j$ th locus across all loci as a result of immigration or recombination will be

$$
\prod_{1}^{j} p_{i j}^{\mathrm{I}} \text { or } \prod_{1}^{j} p_{i j}^{\mathrm{R}}
$$

respectively. For example, the probability that a novel genotype with allele 1 at all loci originated by immigration will be the product of the frequency of allele 1 for every locus in the source population,

$$
p_{11}^{\mathrm{I}} \cdot p_{12}^{\mathrm{I}} \cdot \ldots \cdot p_{1 j}^{\mathrm{I}}
$$

The allele frequencies for the immigration model were based on the allele frequencies in the naturally infected control plots. Similarly, the probability that a novel genotype with allele 1 at all loci originated by recombination will be the product of the frequency of allele 1 for every locus in the population of potential parents,

$$
p_{11}^{\mathrm{R}} \cdot p_{12}^{\mathrm{R}} \cdot \ldots \cdot p_{1 j}^{\mathrm{R}}
$$

The allele frequencies for the recombination model were estimated using allele frequencies calculated from inoculated plots in the previous collection. For example, the absolute probability of occurrence for each novel genotype in the late-season collection was estimated using allele frequencies of all isolates in the inoculated plots in the mid-season collection.

The relative probability of a novel genotype having the $i$ th allele at the $j$ th locus resulting from recombination in the population will be

$$
F_{\mathrm{R}}=\prod_{1}^{j} p_{i j}^{\mathrm{R}} /\left(\prod_{1}^{j} p_{i j}^{\mathrm{I}}+\prod_{1}^{j} p_{i j}^{\mathrm{R}}\right)
$$

Similarly, the relative probability of a novel genotype having the $i$ th allele at the $j$ th locus resulting from immigration in the population will be

$$
F_{\mathrm{I}}=\prod_{1}^{j} p_{i j}^{\mathrm{I}} /\left(\prod_{1}^{j} p_{i j}^{\mathrm{I}}+\prod_{1}^{j} p_{i j}^{\mathrm{R}}\right)
$$

Isolates with relative probabilities of being recombinants or immigrants $\geq 90 \%$ were assigned to categories "recombinant" and "immigrant", respectively. Isolates with probabilities $<90 \%$ were assigned to the category "uncertain" to indicate their lower probability of being either an immigrant or a recombinant.

Our expectation was that ascospore showers that infected the control plots were likely to infect the inoculated plots equally. This assumption is supported by previous experiments showing that the natural $M$. graminicola population was stable at this site over the course of the growing season and across years (5).

Simulation of probability model. To test the ability of this probability method to differentiate between immigrants and recombinants, we conducted a simulation based on random sampling from an artificial population of recombinants and immigrants. The haplotypes of the recombinants in the simulated population were based on the alleles present in the first collection from the inoculated plots. All possible 2,304 haplotypes were generated by joining the alleles for each of the nine loci in all possible combinations. The expected incidence of each haplotype in a sample of 10,000 individuals was calculated as the product of the allele frequencies multiplied by 10,000. For example, consider haplotype 112333263 , which had allele " 1 " at the locus 1 , allele "1" at locus 2 , allele "2" at locus 3, etc. The frequencies of alleles 1, 1, 2, 3, 3, 3, 2,6 , and 3 were $1.0,1.0,0.2,0.4,0.4,0.1,0.5,0.4$, and 0.7 , respectively, in the first collection from the inoculated plots. Following random mating, the expected frequency (absolute probability of occurrence) of the 112333263 haplotype is $4.48 \times 10^{-4}$, and this haplotype would be found four times in the simulated recombinant population. Haplotypes with expected occurrence lower than 0.50 were not included in the simulated population. As a result, a total of 9,813 individuals representing 726 haplotypes was present in the simulated recombinant population. All of these individuals were marked with an R.

The simulated immigrant population was generated in an identical manner based on the alleles and allele frequencies present in the noninoculated plots. In total, 34,560 haplotypes were generated. After removing haplotypes with an expected occurrence lower than 0.50 , a total of 9,325 individuals distributed among 2,347 haplotypes remained in the simulated immigrant population. All of these individuals were marked with an I.

The simulated immigrant and recombinant populations were mixed to form a population of 19,138 individuals. From this mixed population, a random sample of 100 individuals was drawn without replacement. The actual (observed) frequencies of recombinants and immigrants in the sample were based on the actual number of individuals in the sample marked with $\mathrm{R}$ and I. The estimated (expected) frequencies of recombinants and immigrants in the sample were based on the probability model. Each individual was assigned an absolute and relative probability of being a recombinant or immigrant based on allele frequencies in the inoculated or control populations, respectively. Individuals with $F_{\mathrm{R}}$ or $F_{\mathrm{I}} \geq 90 \%$ were placed in the categories recombinant or immigrant, respectively. All other individuals were placed in the uncertain category. The estimated percentage of individuals in the recombinant and immigrant categories based on relative probabilities was compared with the actual percentage in the sample. Estimated and actual frequencies of recombinants and immigrants were calculated for 100 random samples from the mixed population. In addition, the number of isolates incorrectly assigned into the recombinant and immigrant categories (i.e., an I isolate with $F_{\mathrm{R}} \geq 90 \%$ or an R isolate with $F_{\mathrm{I}} \geq 90 \%$ ) was tabulated for each sample. 


\section{RESULTS}

A total of 1,410 M. graminicola isolates was analyzed using the RFLP markers. From the inoculated plots, 582 isolates were sampled during the early season, 183 from the mid-season, and 600 from the late season. Forty-five isolates were sampled from the control plots. The frequency of novel isolates in the inoculated plots increased from 3\% in the early-season to 39 and $34 \%$ in the mid- and late-season collections, respectively. Pairwise differences in frequency were significant, except between the mid- and lateseason collections. None of the 10 inoculated isolates were found in the collections made from the control plots.

In collections from the inoculated plots, only novel isolates were included in estimates of allele frequencies used for contingency chi-squared tests and calculations of gametic disequilibrium. Because the frequency of novel isolates was very small (3\%) in the early-season collection, only the control plots and mid- and lateseason collections from the inoculated plots were characterized for gametic disequilibrium. Alleles with frequencies greater than 0.05 in these three collections are shown in Table 1. Some allele frequencies differed significantly among the collections, e.g., allele 3 of the pSTS197-PstI locus had frequencies of $0.045,0.171$, and 0.239 in the control, mid-, and late-season collections, respectively. Some rare alleles (e.g., allele 21 at locus pSTL31-PstI) appeared either in the control plots or the inoculated plots, but not both. All isolates had the same allele at the pSTS192B-PstI locus, so this locus was not included in any of the analyses. Allele frequencies at two out of the eight remaining loci differed significantly between the control plots and the mid-season inoculated plots, and three out of eight loci had significantly different allele frequencies between the control plots and the late-season inoculated plots (Table 2).

Table 3 summarizes the analyses of multilocus associations among RFLP loci in the $M$. graminicola collections. In the collection from the control plots, the observed variance in heterozygosity $\left(S_{k}^{2}\right)$ did not exceed either L value. $S_{k}^{2}$ was significantly higher than expected in the collections from the inoculated plots.

TABLE 1. Allele frequencies for restriction fragment length polymorphism (RFLP) loci in Mycosphaerella graminicola collections from the control and mid- and late-season inoculated plots

\begin{tabular}{|c|c|c|c|c|}
\hline RFLP locus & Allele $\mathrm{a}^{\mathrm{a}}$ & Control & Mid-season $^{\mathrm{b}}$ & Late season ${ }^{\mathrm{b}}$ \\
\hline \multirow[t]{2}{*}{ pSTS192A-PstI } & 1 & 0.953 & 0.895 & 0.949 \\
\hline & 2 & 0.047 & 0.053 & 0.036 \\
\hline \multirow[t]{3}{*}{ pSTS43-PstI } & 1 & 0.659 & 0.765 & 0.727 \\
\hline & 2 & 0.273 & 0.148 & 0.222 \\
\hline & 3 & 0.068 & 0.062 & 0.040 \\
\hline \multirow[t]{3}{*}{ pSTS2-PstI } & 1 & 0.810 & 0.663 & 0.683 \\
\hline & 2 & 0.024 & 0.048 & 0.075 \\
\hline & 3 & 0.143 & 0.205 & 0.216 \\
\hline \multirow[t]{3}{*}{ pSTS197-PstI } & 1 & 0.591 & 0.573 & 0.660 \\
\hline & 2 & 0.364 & 0.195 & 0.101 \\
\hline & 3 & 0.045 & 0.171 & 0.239 \\
\hline \multirow[t]{5}{*}{ pSTL53-PstI } & 1 & 0.465 & 0.513 & 0.290 \\
\hline & 2 & 0.023 & 0.075 & 0.080 \\
\hline & 3 & 0.070 & 0.038 & 0.160 \\
\hline & 5 & 0.047 & 0.063 & 0.010 \\
\hline & 6 & 0.209 & 0.188 & 0.355 \\
\hline \multirow{2}{*}{ pSTS14-PstI } & 1 & 0.818 & 0.829 & 0.723 \\
\hline & 2 & 0.182 & 0.171 & 0.272 \\
\hline \multirow[t]{5}{*}{ pSTL31-PstI } & 1 & 0.721 & 0.580 & 0.527 \\
\hline & 2 & 0.093 & 0.210 & 0.241 \\
\hline & 3 & 0.000 & 0.074 & 0.030 \\
\hline & 6 & 0.093 & 0.074 & 0.123 \\
\hline & 21 & 0.070 & 0.000 & 0.005 \\
\hline \multirow[t]{2}{*}{ pSTL10-PstI } & 1 & 0.628 & 0.675 & 0.628 \\
\hline & 3 & 0.279 & 0.289 & 0.266 \\
\hline
\end{tabular}

a Alleles with frequencies lower than 0.05 in all three collections are not shown.

b Only novel isolates were used to calculate allele frequencies for the midand late-season collections.
When all allele pairs were used in locus-by-locus measures of gametic disequilibrium, the number of locus pairs showing significant deviations from equilibrium was 1,14 , and 10 out of 28 comparisons for collections from the control (Table 4) and mid- (Table 5) and late-season (Table 6) inoculated plots, respectively. When pooled allele frequencies were used, the number of locus pairs in gametic disequilibrium increased to 3 for the collection from the control plots and decreased to 11 and 8 for collections from the mid- and late-season inoculated plots, respectively (Tables 4 to 6).

The locus pairs at gametic disequilibrium after excluding rare allele pairs were analyzed further using allele-by-allele comparisons. Allele pairs occurring at significantly higher-than-expected frequencies and putative parental isolates are shown in Table 7. In the mid-season collection, 30 out of 104 comparisons showed significant disequilibrium. Nine of these thirty allele pairs occurred at significantly higher-than-expected frequencies. In the late-season collection, 22 out of 129 comparisons showed significant disequilibrium. Ten of these twenty-two allele pairs occurred at significantly higher-than-expected frequencies. In both collections, the majority of allele pairs occurring at higher-than-expected frequencies corresponded to allele pairs present among the 10 inoculated isolates.

The relative probabilities of occurrence for each novel genotype under the models of immigration or recombination among the 10 inoculated isolates are shown in Figure 1. In the mid-season collection, 32 novel isolates had complete data for this analysis. Among these isolates, 20 had $\geq 90 \%$ relative probability of being recombinants or immigrants. Four $(20 \%)$ of the twenty isolates were assigned to the recombinant category and sixteen $(80 \%)$ were assigned to the immigrant category. Twelve isolates were placed in the uncertain category. In the late-season collection, 155 novel isolates had complete data for this analysis. Among these isolates, 56 had $\geq 90 \%$ relative probability of being recombinants or immigrants. Thirty-nine (70\%) of the fifty-six isolates were assigned to the recombinant category and seventeen $(30 \%)$ were assigned to the immigrant category. Ninetynine isolates were placed in the uncertain category.

TABLE 2. Contingency chi-squared tests for homogeneity in allele frequencies among novel isolates in the Mycosphaerella graminicola collections from the control and inoculated plots

\begin{tabular}{lcc}
\hline $\begin{array}{l}\text { Restriction fragment length } \\
\text { polymorphism locus }\end{array}$ & $\begin{array}{c}\text { Control vs. } \\
\text { mid-season }\end{array}$ & $\begin{array}{c}\text { Control vs. } \\
\text { late season }\end{array}$ \\
\hline pSTS192A-PstI & $2.32(2)^{\mathrm{b}}$ & $0.01(1)$ \\
pSTS43-PstI & $3.85(3)$ & $1.69(3)$ \\
pSTS2-PstI & $3.44(3)$ & $3.09(3)$ \\
pSTS197-PstI & $9.44(3)^{*}$ & $23.17(2)^{* *}$ \\
pSTL53-PstI & $9.48(7)$ & $28.15(7)^{* *}$ \\
pSTS14-PstI & $0.02(1)$ & $1.82(2)$ \\
pSTL31-PstI & $12.97(5)^{*}$ & $18.00(4)^{* *}$ \\
pSTL10-PstI & $1.75(2)$ & $0.07(2)$ \\
\hline
\end{tabular}

a $*=$ Significant at $p=0.05$. $* *=$ Significant at $p=0.01$.

$\mathrm{b}$ The degrees of freedom for each test are shown in parentheses.

TABLE 3. Multilocus associations among restriction fragment length polymorphism (RFLP) loci in the Mycosphaerella graminicola collections from the control and mid- and late-season inoculated plots ${ }^{\mathrm{a}}$

\begin{tabular}{lcrccccc}
\hline Population & $m^{\mathrm{b}}$ & \multicolumn{1}{c}{$n^{\mathrm{c}}$} & $h^{\mathrm{d}}$ & $u(2)^{\mathrm{e}}$ & $L_{1}{ }^{\mathrm{f}}$ & $L_{2}{ }^{\mathrm{g}}$ & $S_{k}^{2 \mathrm{~h}}$ \\
\hline Control plots & 8 & 44 & 0.4273 & 1.709 & 2.396 & 2.727 & 0.803 \\
Mid-season & 8 & 83 & 0.4670 & 1.789 & 2.132 & 2.577 & $2.643^{* * \mathrm{i}}$ \\
Late season & 8 & 203 & 0.4850 & 1.734 & 2.055 & 2.219 & $2.078^{*}$ \\
\hline
\end{tabular}

a Data based on the measure of Brown et al. (2).

${ }^{\mathrm{b}} m=$ Number of RFLP loci.

${ }^{\mathrm{c}} n=$ Population size.

d $h=$ Mean single-locus gene diversity.

e $u(2)=$ Expected central moment under null hypothesis.

f $L_{1}=$ Upper $95 \%$ confidence limit.

g $L_{2}=$ Upper $99 \%$ confidence limit.

h $S_{k}^{2}=$ Observed variance of the number of heterozygous comparisons.

i $*=$ Indicates $S_{k}^{2}$ exceeds $L_{1} \cdot{ }^{* *}=$ Indicates $S_{k}^{2}$ exceeds $L_{2}$. 
Results from the computer simulation of the probability method are shown in Figure 2. There was a significant correlation $(r=0.78)$ between the observed frequencies of recombinants and immigrants in the random sample and the expected frequencies calculated using the probability method described above (Fig. 2). On average, $2 \%$ of the isolates were incorrectly placed into the recombinant and $4 \%$ were incorrectly placed into the immigrant categories. As a further test of the reliability of the probability method, 10 isolates were selected randomly from the late-season collection for each of the three categories, and their DNA fingerprints were compared with those of the 10 inoculated isolates. By this comparison, we hoped to directly validate the probability method by identifying recombinant isolates that shared fragments found in the DNA fingerprints of putative parents (Fig. 3). We found no putative recombinants among the 10 isolates chosen from the immigrant category, while three and nine putative recombinants were identified among isolates chosen from the uncertain and recombinant categories, respectively.

\section{DISCUSSION}

Neutral DNA markers were used to estimate the relative contributions of immigration, sexual reproduction, and asexual reproduction to the genetic structure of a $M$. graminicola population during the course of a field epidemic. Our results indicate that the importance of sexual reproduction increased and the role of immigration decreased during a winter wheat growing season. At the time of the second collection, we estimate that $8 \%$ of the population originated from recombination, $31 \%$ originated by immigration, and $61 \%$ were the asexual descendants of the original 10 inoculated isolates. By the time of our third collection, we estimate that $24 \%$ of the population originated from recombination, $10 \%$ originated from immigration, and $66 \%$ by asexual reproduction.

In collections from the inoculated plots, the proportion of novel isolates increased from $3 \%$ in the early season to $39 \%$ in the midseason. We believe that few novel genotypes were detected in the inoculated plots early in the season because the lesions originating from the natural ascospore cloud were vastly outnumbered by the

TABLE 4. Pairwise measures ${ }^{\mathrm{a}}$ of gametic disequilibrium between restriction fragment length polymorphism (RFLP) loci in the collection of Mycosphaerella graminicola from the control plots

\begin{tabular}{lcccccccc}
\hline & \multicolumn{9}{c}{ RFLP loci } \\
\cline { 2 - 8 } RFLP loci & pSTS192A & pSTS43 & pSTS2 & pSTS197 & pSTL53 & pSTS14 & pSTL31 & pSTL10 \\
\hline pSTS192A & $\ldots$ & $3.08(2)$ & $0.43(3)$ & $3.78(2)$ & $9.64(8)$ & $2.02(1)$ & $7.07(4)$ \\
pSTS43 & $3.08(2)$ & $\ldots$ & $5.61(6)$ & $3.05(4)$ & $10.79(16)$ & $3.03(2)$ & $7.28(8)$ & $13.98(5)$ \\
pSTS2 & $0.43(2)$ & $5.61(4)$ & $\ldots$ & $8.32(6)$ & $24.48(24)$ & $6.44(3)$ & $2.77(12)$ & $44.10(15) *$ \\
pSTS197 & $3.78(2)$ & $3.05(4)$ & $8.23(4)$ & $\ldots$ & $7.29(7)$ & $1.05(2)$ & $5.86(18)$ & $5.58(8)$ \\
pSTL53 & $9.64(3)^{* b}$ & $6.06(6)$ & $0.15(2)$ & $1.33(2)$ & $\ldots$ & $8.13(9)$ & $26.78(32)$ & $39.21(40)$ \\
pSTS14 & $2.02(1)$ & $3.03(2)$ & $6.44(3)$ & $1.05(2)$ & $8.13(3)^{*}$ & $\ldots$ & $7.00(4)$ & $6.91(5)$ \\
pSTL31 & $3.14(1)$ & $2.22(2)$ & $1.21(4)$ & $4.19(4)$ & $5.07(4)$ & $0.03(1)$ & $\ldots$ & $7.98(10)$ \\
pSTL10 & $0.98(2)$ & $9.67(4)^{*}$ & $6.59(4)$ & $2.21(4)$ & $11.44(6)$ & $0.66(2)$ & $1.80(2)$ & $\ldots$ \\
\hline
\end{tabular}

a Numbers above and below the diagonal are chi-squared values and their corresponding degrees of freedom (in parentheses) before and after rare alleles (alleles present at frequencies less than 0.10 ) were pooled into a single category, respectively.

$\mathrm{b} *=$ Significant at $p=0.05$.

TABLE 5. Pairwise measures ${ }^{\mathrm{a}}$ of gametic disequilibrium between restriction fragment length polymorphism (RFLP) loci in the collection of Mycosphaerella graminicola from the mid-season inoculated plots

\begin{tabular}{|c|c|c|c|c|c|c|c|c|}
\hline \multirow[b]{2}{*}{ RFLP loci } & \multicolumn{8}{|c|}{ RFLP loci } \\
\hline & pSTS192A & pSTS43 & pSTS2 & pSTS197 & pSTL53 & pSTS14 & pSTL31 & pSTL10 \\
\hline pSTS192A & & $28.16(12)^{* * b}$ & $114.74(20)^{* *}$ & 7.99 (12) & $54.19(36)^{*}$ & $4.97(4)$ & $83.23(28)^{* *}$ & $15.32(12)$ \\
\hline pSTS2 & $13.58(2)^{* *}$ & $11.61(6)$ & & $11.59(18)$ & $71.20(54)$ & $17.66(4)^{* *}$ & $36.23(35)$ & $107.87(18)^{* * *}$ \\
\hline pSTS197 & $3.04(3)$ & $8.44(6)$ & $6.96(6)$ & $\ldots$ & $18.48(27)$ & $13.11(3)^{*}$ & $42.42(21)^{*}$ & $8.61(9)$ \\
\hline pSTL53 & $0.70(2)$ & $0.39(4)$ & $9.78(4) *$ & $8.20(6)$ & & $4.67(9)$ & $85.20(63)^{*}$ & $44.03(21)^{*}$ \\
\hline pSTS14 & $0.50(1)$ & $18.72(2) * * *$ & $6.41(2)^{*}$ & $13.11(3)^{* *}$ & $1.18(2)$ & $\ldots$ & $14.75(7)^{*}$ & $7.57(3)$ \\
\hline
\end{tabular}

${ }^{a}$ Numbers above and below the diagonal are chi-square values and their corresponding degrees of freedom (in parentheses) before and after rare alleles (alleles present at frequencies less than 0.10 ) were pooled into a single category, respectively.

$\mathrm{b} *=$ Significant at $p=0.05 . * *=$ Significant at $p=0.01 . * * *=$ Significant at $p=0.001$

TABLE 6. Pairwise measures ${ }^{\mathrm{a}}$ of gametic disequilibrium between restriction fragment length polymorphism (RFLP) loci in the collection of Mycosphaerella graminicola isolates from the late-season inoculated plots

\begin{tabular}{lcccccccc}
\hline & \multicolumn{7}{c}{ RFLP loci } \\
\cline { 2 - 8 } RFLP loci & pSTS192A & pSTS43 & pSTS2 & pSTS197 & pSTL53 & pSTS14 & pSTL31 & pSTL10 \\
\hline pSTS192A & $\ldots$ & $4.44(9)$ & $50.09(15)^{* * b}$ & $1.80(6)$ & $11.10(30)$ & $5.73(6)$ & $115.08(24)^{* *}$ & $26.70(21)$ \\
pSTS43 & $1.48(2)$ & $\ldots$ & $6.58(15)$ & $5.86(6)$ & $37.62(27)$ & $12.64(6)^{*}$ & $36.03(24)$ & $45.60(24)^{*}$ \\
pSTS2 & $15.34(2)^{* * *}$ & $6.15(4)$ & $\ldots$ & $19.61(10)^{*}$ & $33.40(50)$ & $18.73(10)^{*}$ & $69.24(40)^{*}$ & $10.40(35)$ \\
pSTS197 & $0.19(2)$ & $4.78(4)$ & $14.04(4) * *$ & $\ldots$ & $23.94(20)$ & $6.77(4)$ & $31.56(16)^{*}$ & $22.20(12) *$ \\
pSTL53 & $6.11(3)$ & $25.56(6)^{* * *}$ & $5.43(6)$ & $8.56(6)$ & $\ldots$ & $213.10(20)^{* * *}$ & $98.76(80)$ & $52.24(80)$ \\
pSTS14 & $3.77(2)$ & $8.08(4)$ & $13.84(4)^{* *}$ & $6.77(4)$ & $9.68(6)$ & $\ldots$ & $20.71(14)$ & $11.87(16)$ \\
pSTL31 & $6.47(3)$ & $3.93(6)$ & $37.34(6)^{* * *}$ & $25.60(6)^{* * *}$ & $30.52(9)^{* * *}$ & $15.99(6)^{*}$ & $\ldots$ & $52.45(64)$ \\
pSTL10 & $0.02(2)$ & $8.00(4)$ & $6.22(6)$ & $1.82(4)$ & $1.72(6)$ & $8.32(4)$ & $1.96(6)$ & $\ldots$ \\
\hline
\end{tabular}

${ }^{a}$ Numbers above and below the diagonal are chi-squared values and their corresponding degrees of freedom (in parentheses) before and after rare alleles (alleles present at frequencies less than 0.10 ) were pooled into a single category, respectively.

$\mathrm{b} *$ Significant at $p=0.05$. $* *=$ Significant at $p=0.01$. $* * *=$ Significant at $p=0.001$. 
lesions originating from the hundreds of millions of pycnidiospores introduced artificially into each plot. The novel genotypes that emerged later in the season could have resulted from ascospores that immigrated from outside of the experimental plots or from sexual reproduction among the isolates within the plots. To differentiate immigrants from recombinants, we compared the genetic structures of $M$. graminicola collections from control plots and inoculated plots in the same field. Our rationale was that the genetic structure of the $M$. graminicola collections would be the same if both had originated from the same ascospore cloud. Statistical differences in the genetic structures of the two populations would imply different origins for the populations, i.e., immigration versus recombination.

Our results support the hypothesis that the M. graminicola isolates in the control plots originated from ascospores unrelated to the 10 inoculated genotypes, while a significant fraction ( $70 \%$ by late season) of the novel isolates in the inoculated plots originated from sexual recombination among the 10 inoculated isolates. This hypothesis was supported by five lines of evidence. (i) Significant differences in allele frequencies were present between collections from the control and inoculated plots. (ii) Alleles at individual RFLP loci were at gametic equilibrium in the control plots, but disequilibrium existed in the inoculated plots. (iii) Most allele pairs that occurred at higher-than-expected frequencies corresponded to allele pairs present among the 10 inoculated isolates. (iv) A large fraction of the novel multilocus haplotypes exhibited a higher probability of originating from recombination than from immigration. (v) Comparisons of DNA fingerprints and single-copy RFLP loci showed that most novel isolates in the recombinant category possessed the same DNA fragments and RFLP alleles, respectively, present in the inoculated isolates (Fig. 3). None of the isolates in the immigrant category had DNA fingerprints or multilocus haplotypes consistent with recombination among the inoculated isolates.

Each novel multilocus haplotype was assigned into an immigrant, recombinant, or uncertain category based on the relative probability of being a recombinant or immigrant (Fig. 1). Some novel isolates could immediately be assigned to the immigrant category because they possessed RFLP alleles found in the control population but not in the 10 inoculated isolates or the previous collection. In the recombinant category, many novel isolates had alleles found at a high frequency in the 10 inoculated isolates and at a low frequency in the control plots. The simulation validated the use of absolute and relative probabilities to differentiate immigrants and recombinants in a field setting. The simulation indicated that the probability method could differentiate between immigrants and recombinants with 96 to $98 \%$ accuracy. The simulation also showed that the estimated frequencies of immigrants and recombinants were highly correlated with the observed frequencies of immigrants and recombinants in each sample.

Each of the three methods used to measure gametic disequilibrium gave the same result. The collection from the control plots was in gametic equilibrium, while the mid- and late-season collections from the inoculated plots had significant levels of gametic disequilibrium. The gametic equilibrium in the control plots supported the hypothesis that the primary inoculum for these plots was ascospores. The disequilibrium among the novel isolates in the inoculated plots was interpreted as evidence for recombination among the inoculated isolates.

Analyses of multilocus associations according to Brown et al. (2) are less influenced by rare allele pairs than the methods proposed by Weir (37). One drawback of Brown's method is that it ignores the behavior of particular allele combinations. Therefore, we further evaluated multilocus associations with the locus-bylocus methods of Weir. Locus-by-locus comparisons also did not provide evidence for meaningful gametic disequilibrium in the control population. The finding of a large number of different genotypes and independent associations among RFLP alleles suggests that these isolates of $M$. graminicola originated from ascospore showers (6).

In collections from the inoculated plots, the variance in the number of loci having different alleles (heterozygous) when two individuals were compared $\left(s_{k}^{2}\right)$ was significantly higher than expected (Table 3). Locus-by-locus comparisons also indicated a strong nonrandom association among many RFLP loci (Tables 5 and 6), suggesting that at least some of these isolates of $M$. graminicola originated from sexual recombination among the 10 inoculated isolates. Allele-by-allele comparisons indicated that 52 out of 233 allele pairs exhibited a significant departure from gametic equilibrium in the mid-season and late-season collections. Sixteen (31\%) of these fifty-two allele pairs corresponded to allele pairs present in the inoculated isolates. The frequency of inoculated isolate M1 decreased significantly during the growing season. Isolate M5 first

TABLE 7. Restriction fragment length polymorphism (RFLP) allele pairs occurring at higher-than-expected frequencies in the mid- and late-season collections of Mycosphaerella graminicola from the inoculated plots

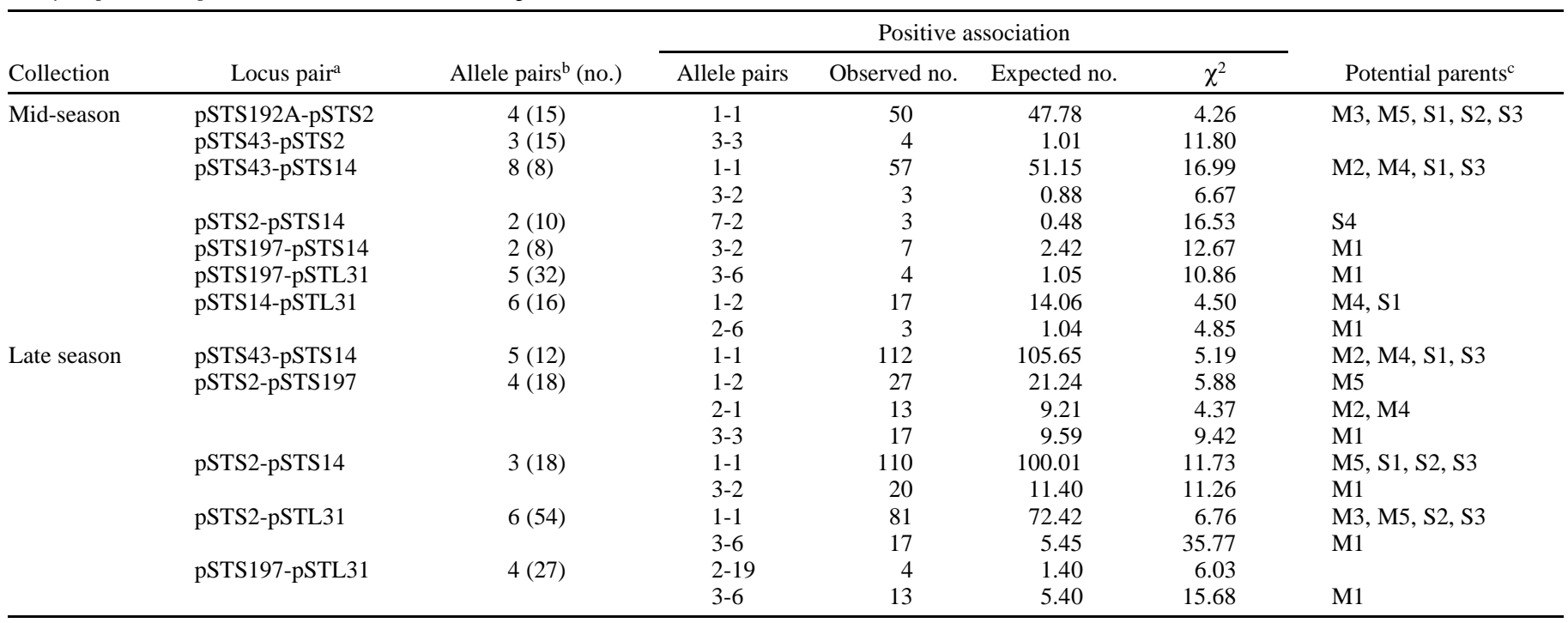

a Pairs of loci with significant departures from gametic equilibrium as measured by locus-by-locus comparison.

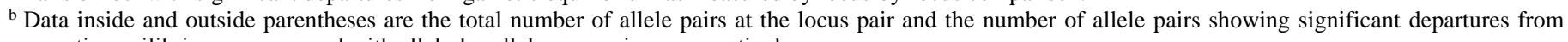
gametic equilibrium as measured with allele-by-allele comparison, respectively.

c The inoculated isolates that possessed the same allele pairs in their multilocus haplotypes. 
increased and later decreased in frequency during the growing season (J. Zhan and B. A. McDonald, unpublished data). We suspect that both of these isolates were involved in sexual reproduction during the course of the epidemic. Indeed, isolates M1 and M5 were the potential parents for $11(58 \%)$ out of 19 allele pairs that occurred at significantly higher-than-expected frequencies.

The mid-season collection had a higher degree of gametic disequilibrium than the late-season collection. The mid-season collec-

\section{A}
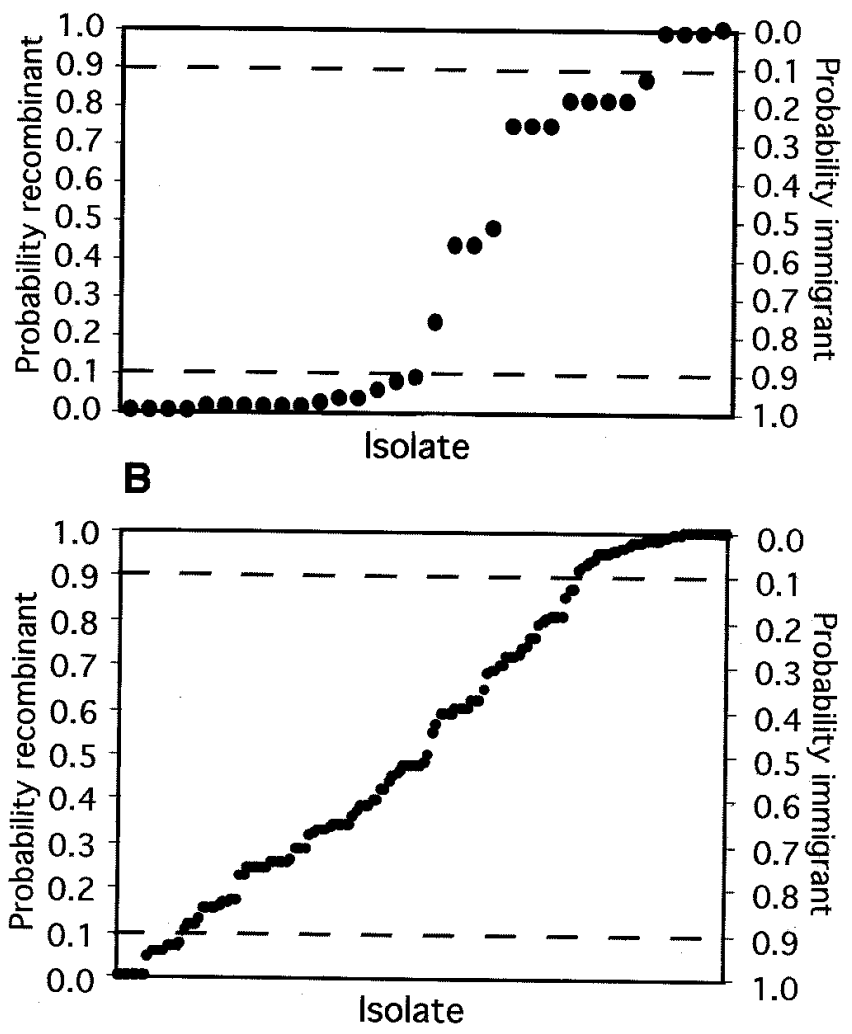

Fig. 1. The relative probability of novel Mycosphaerella graminicola isolates originating from immigration or sexual recombination in the $\mathbf{A}$, mid-season or B, late-season collections. Novel isolates with $\geq 90 \%$ probability of being immigrants or recombinants are present beyond the $90 \%$ dashed lines. Novel isolates with lower probability of being recombinants or immigrants were placed in the uncertain category between the dashed lines.

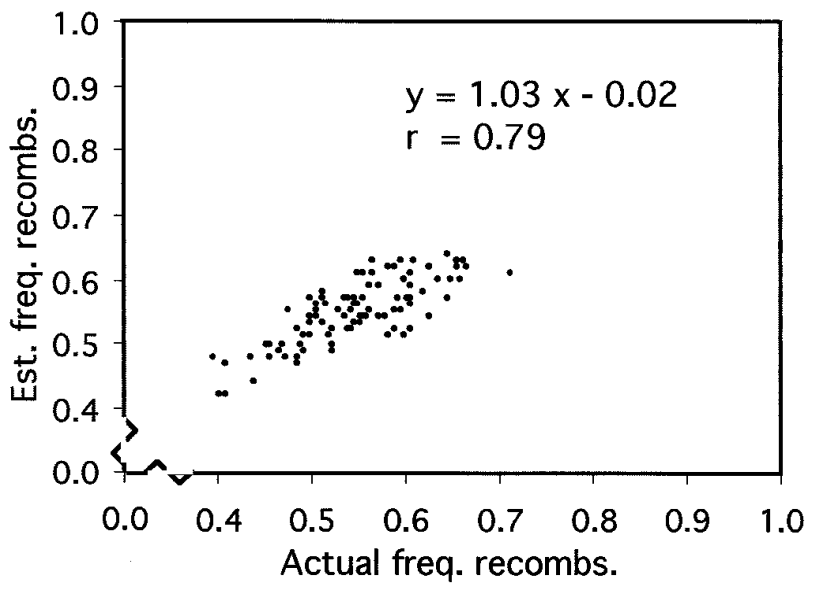

Fig. 2. Simulation of probability method used to differentiate recombinants and immigrants. Actual frequencies of recombinants in 100 random draws from a mixed population of immigrants and recombinants were compared with the estimated frequencies calculated using the probability method described in the text. tion departed from gametic equilibrium at $p=0.01$ compared with significance at $p=0.05$ in the late-season collection (Table 3 ). The decline in gametic disequilibrium during this period probably was due to continuous intermating among the novel or inoculated isolates rather than immigration of new ascospores. In the mid-

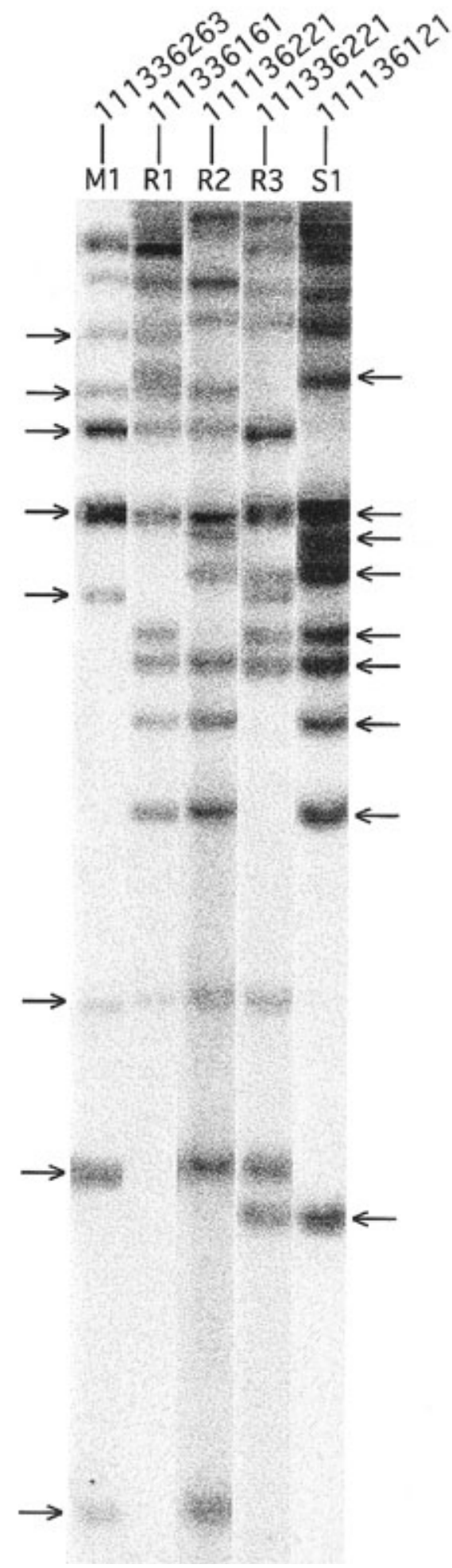

Fig. 3. DNA fingerprints of inoculated Mycosphaerella graminicola isolates $\mathrm{M} 1$ and S1 and putative recombinant progeny R1, R2, and R3. DNA fingerprint probe pSTL70 was hybridized to DNA digested with PstI. Multilocus haplotypes based on eight other probes that hybridized to nine independent loci are indicated above each lane. Novel isolates R1, R2, and R3 have reassorted DNA fingerprint fragments (indicated with arrows) and restriction fragment length polymorphism alleles (multilocus haplotypes above each lane) in common with inoculated isolates M1 and S1. 
season, we estimated that immigrants accounted for $80 \%$ of the novel isolates. This proportion decreased to $30 \%$ in the late season. This suggests that immigration had a stronger impact on the genetic structure of $M$. graminicola populations in the early and mid-season compared with the late season.

The statistical power to detect disequilibrium between alleles of different loci is affected by sample sizes and allele frequencies. The power decreases when sample sizes are small, and it decreases further when loci have low gene diversity $(11,39)$. Unless allele frequencies are intermediate and gametic disequilibrium is strong, a relatively large sample size $(>50)$ is needed to detect a nonrandom association (1). Therefore, the inferences obtained from small sample sizes might not be robust (25). In our experiment, the sample size used to calculate gametic disequilibrium was only 45 for the population made from the control plots. This sample size was suboptimal for this type of analysis, but we do not believe that it affected our conclusions. With a much larger sample size of 617 isolates from the same experiment station 4 years earlier, we did not detect any gametic disequilibria in a naturally inoculated field (6).

Our experiment illustrates a general method that may be used to estimate the relative contributions of immigration, sexual reproduction, and asexual reproduction in field populations of pathogenic fungi. The application of this method requires some knowledge of the genetic structure of local natural populations and polymorphic, reproducible, neutral genetic markers. The power of the experiment is optimized by choosing inoculated isolates with rare alleles not present in the local population. Alternatively, the inoculated collection of isolates should have allele frequencies that differ significantly compared with the natural local population.

\section{ACKNOWLEDGMENTS}

This project was supported by National Science Foundation grant number DEB-9306377. We thank M. Hoffer for her contributions to the field aspects of this project.

\section{LITERATURE CITED}

1. Brown, A. H. D. 1975. Sample sizes required to detect linkage disequilibrium between two or three loci. Theor. Popul. Biol. 8:184-201.

2. Brown, A. H. D., Feldman, M. W., and Nevo, E. 1980. Multilocus structure of natural populations of Hordeum spontaneum. Genetics 96:523-536.

3. Brown, J. K. M., and Wolfe, M. S. 1990. Structure and evolution of a population of Erysiphe graminis f. sp. hordei. Plant Pathol. 39:376-390.

4. Burdon, J. J., and Roelfs, A. P. 1985. The effect of sexual and asexual reproduction on the isozyme structure of populations of Puccinia graminis. Phytopathology 75:1068-1073.

5. Chen, R. S., Boeger, J. M., and McDonald, B. A. 1994. Genetic stability in a population of a plant pathogenic fungus over time. Mol. Ecol. 3:209-218.

6. Chen, R. S., and McDonald, B. A. 1996. Sexual reproduction plays a major role in the genetic structure of populations of the fungus $M y$ cosphaerella graminicola. Genetics 142:1119-1127.

7. Everitt, B. S. 1977. The Analysis of Contingency Tables. John Wiley \& Sons, Inc., New York.

8. Eyal, Z. 1981. Integrated control of Septoria diseases of wheat. Plant Dis. 65:763-768.

9. Eyal, Z., Scharen, A. L., Huffman, M. D., and Prescott, J. M. 1985. Global insights into virulence frequencies of Mycosphaerella graminicola. Phytopathology 75:1456-1462.

10. Eyal, Z., and Ziv, O. 1974. The relationship between epidemics of Septoria leaf blotch and yield losses in spring wheat. Phytopathology 64:1385-1389.

11. Fu, Y. X., and Arnold, J. 1992. A table of exact sample size for use with Fisher's exact test for $2 \times 2$ tables. Biometrics 48:1103-1112.

12. Hunter, T., Coker, R. R., and Royle, D. J. 1997. The sexual phase, Mycosphaerella graminicola, in epidemics of leaf blotch in winter wheat (Abstr.) Page 23 in: 15th Long Ashton International Symposium-Understanding Pathosystems: A Focus on Septoria. IACR-Long Ashton, Bristol, England.

13. Kema, G. H. J., Verstappen, E. C. P., Todorova, M., and Waalwijk, C. 1996. Successful crosses and molecular tetrad and progeny analysis demonstrate heterothallism in Mycosphaerella graminicola. Curr. Genet. 30:252-258.
14. King, J. E., Cook, R. J., and Melville, S. C. 1983. A review of Septoria diseases of wheat and barley. Ann. Appl. Biol. 103:345-373.

15. Koenig, R. L., Ploetz, R. C., and Kistler, H. C. 1997. Fusarium oxysporum f. sp. cubense consists of a small number of divergent and globally distributed clonal lineages. Phytopathology 87:915-923.

16. Leath, S., Scharen, A. L., Lund, R. E., and Dietz-Holmes, M. E. 1993 Factors associated with global occurrences of Septoria nodorum blotch and Septoria tritici blotch of wheat. Plant Dis. 77:1266-1270.

17. Leung, H., Nelson, R. J., and Leach, J. E. 1993. Population structure of plant pathogenic fungi and bacteria. Pages 157-205 in: Advances in Plant Pathology, Vol. 10. J. H. Andrews and I. C. Tommerup, eds. Academic Press, New York.

18. McDonald, B. A., and Martinez, J. P. 1990. DNA restriction fragment length polymorphisms among Mycosphaerella graminicola (anamorph Septoria tritici) isolates collected from a single wheat field. Phytopathology 80:1368-1373.

19. McDonald, B. A., and Martinez, J. P. 1990. Restriction fragment length polymorphisms in Septoria tritici occur at a high frequency. Curr. Genet. $17: 133-138$.

20. McDonald, B. A., and Martinez, J. P. 1991. DNA fingerprinting of the plant pathogenic fungus Mycosphaerella graminicola (anamorph Septoria tritici). Exp. Mycol. 15:146-158.

21. McDonald, B. A., and Martinez, J. P. 1991. Chromosome length polymorphisms in a Septoria tritici population. Curr. Genet. 19:265-271.

22. McDonald, B. A., and McDermott, J. M. 1993. Population genetics of plant pathogenic fungi. Bioscience 43:311-319.

23. McDonald, B. A., Mundt, C. C., and Chen, R. S. 1996. The role of selection on the genetic structure of pathogen populations: Evidence from field experiments with Mycosphaerella graminicola on wheat. Euphytica 92:73-80.

24. McDonald, B. A., Zhan, J., Yarden, O., Hogan, K., Garton, J., and Pettway, R. E. 1998. The population genetics of Mycosphaerella graminicola and Phaeosphaeria nodorum. Pages 44-69 in: Septoria on Cereals: A Study of Pathosystems. J. A. Lucas, P. Bowyer, and H. M. Anderson, eds. CAB International, Wallingford, United Kingdom.

25. Milgroom, M. G. 1996. Recombination and the multilocus structure of fungal populations. Annu. Rev. Phytopathol. 34:457-477.

26. Mundt, C. C., Hoffer, M. E., Ahmed, H. U., Coakley, S. M., DiLeone, J. A., and Cowger, C. 1998. Population genetics and host resistance. Pages 115-130 in: Septoria on Cereals: A Study of Pathosystems. J. A. Lucas, P. Bowyer, and H. M. Anderson, eds. CAB International, Wallingford, United Kingdom.

27. Punja, Z. K., Grogan, R. G., and Adams, G. C. 1982. Influence of nutrition, environment and the isolate on basidiocarp formation, development and structure in Athelia (Sclerotium) rolfsii. Mycologia 74:917-926.

28. Rillo, A. O., and Caldwell, R. M. 1966. Inheritance of resistance to Septoria tritici in Triticum aestivum subsp. vulgare "Bulgaria 88". (Abstr.) Phytopathology 56:897.

29. Roelfs, A. P., and Groth, J. V. 1980. A comparison of virulence phenotypes in wheat stem rust populations reproducing sexually and asexually. Phytopathology 70:855-862.

30. Rosielle, A. A., and Brown, A. G. P. 1979. Inheritance, heritability and breeding behavior of three sources of resistance to Septoria tritici in wheat. Euphytica 28:385-392.

31. Royle, D. J., Shaw, M. W., and Cook, R. J. 1986. The natural development of Septoria nodorum and S. tritici in some winter wheat crops in Western Europe, 1981-83. Plant Pathol. 35:466-476.

32. Shaner, G., Finney, R. E., and Paterson, F. L. 1975. Expression of effectiveness of resistance in wheat to Septoria leaf blotch. Phytopathology 65:761-766.

33. Shaw, M. W., and Royle, D. J. 1989. Airborne inoculum as a major source of Septoria tritici (Mycosphaerella graminicola) infections in winter wheat crops in the UK. Plant Pathol. 38:35-43.

34. Slatkin, M. 1985. Gene flow in natural populations. Annu. Rev. Ecol. Syst. 16:393-410.

35. Slatkin, M. 1987. Gene flow and the geographic structure of natural populations. Science 236:787-792.

36. Van Ginkel, M., and Scharen, A. L. 1988. Host-pathogen relationships of wheat and Septoria tritici. Phytopathology 78:762-766.

37. Weir, B. S. 1990. Genetic Data Analysis. Sinauer Associates, Sunderland, MA.

38. Wilson, R. E. 1979. Resistance to Septoria tritici in two wheat cultivars determined by independent, single dominant genes. Aust. Plant Pathol. 8:16-18.

39. Zapata, C., and Alvarez, G. 1993. On the detection of non-random associations between DNA polymorphisms in natural populations of Drosophila. Mol. Biol. Evol. 10:823-842. 